\title{
Legitimidad y modernización de la Administración Pública ${ }^{1}$
}

\section{Ernesto Carrillo *}

\section{Introducción}

El propósito de este artículo es llevar a cabo un análisis de los siguientes problemas de investigación: ¿en qué medida las reformas del sistema políico-administrativo y la modemización de la Administración pública influyen sobre la legitimidad de la Administración? y ¿en qué grado los rendimientos de ésta contribuyen a dotar de eficacia al sistema político?

El interés de estas cuestiones no es sólo teórico —de hecho, son asuntos de la máxima relevancia desde la perspectiva de la Ciencia Política y de la Administración- sino que también ofrecen información sobre aspectos a considerar en relación con la formulación de las políticas de reforma y modernización de las Administraciones públicas.

Se trata en ambos casos de problemas muy vastos sobre los que este trabajo proporciona una primera indagación empírica para el caso español, complementaria - por lo que respecta a la Administración- de los abundantes y excelentes estudios existentes en nuestro país sobre la cultura política. De hecho, la evidencia empírica que se aporta está basada principalmente en datos de opinión pública lo cual supone la contemplación de una dimensión crítica del problema, pero también implica una limitación del estudio. Entiéndanse, pues, estas notas más que como respuestas definitivas a las preguntas arriba formuladas, como un intento de avanzar en la indagación de ambos problemas de investigación.

El artículo se inicia con unas breves reflexiones teóricas sobre el problema de la legitimidad, para posteriormente analizar el impacto de las grandes reformas del sistema político-administrativo en España, su grado de aceptación en términos de opinión y los cambios en la percepción de eficacia de los servicios públicos. A continuación se analizará lo que se denomina presunción de ineficiencia - una de las constantes respecto de la imagen de la Administración- para poner el énfasis en lo que es quizá el aspecto más relevante: los problemas de legitimidad de las Administraciones públicas. Asimismo, se harán consideraciones sobre las orientaciones de las políticas de modemización y su contribución a la legitimidad de la Administración pública.

\section{La legitimidad de la Administración Pública}

Un conocido trabajo de LIPSET (1987) subraya que la estabilidad de cualquier democracia depende, entre otros factores, de la legitimidad y de la eficacia de su sistema político. Para este autor, la slegitimidad implica la capacidad del sistema para engendrar y mantener la creencia de que las instituciones políticas existentes son las más apropiadas para la sociedad, mientras que la reficacia significa verdadera actuación, el grado en que el sistema satisface las funciones básicas tales como las consideran la mayoría de la población y grupos tan poderosos en ella como son las altas finanzas o las fuerzas armadas. Al margen de consideraciones sobre la concepción elitista de la política que estas definiciones puedan contener, o de las limitaciones conceptuales que supone vincular la noción de legitimidad a la -creencia. en la legitimidad, o el hecho de que la eficacia sea también uno de los componentes de la legitimidad ${ }^{2}$, lo cierto es que esta distinción resulta de utilidad a la hora de analizar el problema de la legitimidad de la Administración pública. 
En otro lugar, y apoyándome en este planteamiento, distinguia dos fuentes de legitimidad de la Administración: la legitimidad institucional que deriva del ajuste del comportamiento de la Administración a un sistema de valores socialmente aceptados sobre cómo debe ser la Administración pública en un Estado social y democrático de Derecho; y la legitimidad por rendimientos, que se obtiene produciendo políticas, bienes y servicios públicos que respondan a criterios de evaluación de lo público socialmente aceptados.

En cualquier caso, y siguiendo el esquema de LIPSET, una de las contribuciones principales del gobierno y la Administración pública a la estabilidad de la democracia es proporcionar eficacia al sistema político mediante las políticas y los servicios públicos. Según afirma este autor, un sistema que fuera legítimo y que, al mismo tiempo, fuera ineficaz de forma persistente, tarde o temprano terminaría por arruinar su legitimidad. Por otra parte, la legitimidad del sistema político lo es también de la Administración, de manera que difícilmente podría existir una Administración pública legítima si el sistema político en su conjunto no gozara de legitimidad.

Paralelamente, las necesidades de legitimación de la Administración pública han ido creciendo con el tiempo -entre otras cosas por la acumulación histórica de criterios en los que se basa la justificación de la Administración- asistiéndose en la actualidad a un cuestionamiento de lo público. La teoría de los fallos del Estado o las posibilidades de separar organizativamente la producción y la provisión de servicios públicos, marcan limitaciones de índole conceptual al alcance del sector público y ofrecen instrumentos técnicos de gestión en virtud de los cuales el sistema político puede garantizar en parte sus necesidades de eficacia sin recurrir a la producción de servicios a través de la Administración pública.

Frente a esta situación, surge la pregunta de en qué basar la legitimidad de la Administración y, más en concreto, cuáles son los criterios de legitimidad. De acuerdo con BeEtham (1991) puede decirse que un poder es legítimo en la medida en que:

Primero, se adecúe a las reglas establecidas, esto es, cuente con validez legal en la adquisición y el ejercicio del poder.

Así, por ejemplo, una Administración que no cumpla las reglas - sean éstas leyes escritas o normas no escritas- del sistema de mérito sería una Administración ilegítima y su gobiemo estaría sometido a crítica por ello. Desde esta perspectiva, una Administración profesional que se aproxime al modelo de dominación racional-legal con cuadro administrativo burocrático formulado por WEBER (1979) en su versión de tipo ideal cumpliría con este criterio de legitimidad en un Estado democrático de Derecho.
Segundo, que las reglas establecidas puedan justificarse por su relación con una serie de creencias compartidas por los dominadores y los subordinados. La justificabilidad de las reglas es un criterio a añadir al anterior, pues éstas no sólo deben disponer de validez legal sino que además deben ser justificables en términos de creencias y valores presentes en una sociedad. De tal forma que el desajuste entre reglas y creencias, así como la ausencia de un marco de creencias compartidas, acabaría produciendo un déficit de legitimidad.

Este requisito supone un nivel de exigencia mucho mayor que el anterior, pues en sistemas político-administrativos en los que el gasto público ronda el 50 por 100 del PIB la acumulación de demandas hacia lo público es considerable tanto en términos de rendimiento -donde las expectativas de valor son frecuentemente contradictorias - como de formas de comportamiento -basadas en sistemas de valores múltiples en los que es imposible la maximización de alguno de ellos sin consecuencias sobre los restantes-. Así, por lo que respecta a los rendimientos se exige que sean justos -en cuanto al sistema de reparto de costes y beneficios-, de calidad -en el sentido de que satisfagan o excedan las expectativas de beneficio de los ciudadanos-, eficaces en sus impactos y eficientes -en lo relativo a la ratio entre impactos y recursos invertidos- ${ }^{3}$. A su vez el sistema de valores respecto al comportamiento de lo público involucra, además de las ideas de legalidad $-\mathrm{y}$ todo lo que ello lleva asociado-, la profesionalidad de los empleados públicos y la separación entre políica y administración, así como muchos otros valores como puedan ser: una noción de equidad y un tipo de alcance en cuanto a la intervención del Estado; además de otros aspectos asociados con la idea de responsabilidad administrativa y el gobierno y la administración democrática - receptividad, rendición de cuentas, participación, comportamiento ético, flexibilidad, competencia, etc.- (Starung, 1986). Ante este cúmulo de criterios no tiene nada de particular que puedan existir déficits de legitimidad en los gobiemos y las Administraciones, así como en el conjunto de lo público.

Tercero, que exista evidencia de aprobación mediante acciones que expresen el consentimiento por parte de los subordinados en la relación de poder considerada. En el caso de que se erosionen las acciones a través de las cuales se exprese el consentimiento tendrá lugar un proceso de deslegitimación.

Las acciones a través de las cuales los ciudadanos expresan su consentimiento adquieren muy diversas formas en lo que respecta al gobierno y la Administración: mediante el pago de impuestos y otras contribuciones obligatorias o voluntarias en fuerza de trabajo; cumpliendo las regulaciones; 
consumiendo servicios públicos; y a través de la participación. En un contexto de deslegitimación encontraríamos fenómenos como las revueltas fiscales, la insumisión, la resistencia masiva al cumplimiento de las regulaciones, el recurso a servicios privados altemativos y/o sustitutorios de los servicios públicos cuando exista la posibilidad de elección, o el rechazo de las oportunidades de participación.

A partir de estas consideraciones veamos cuál es la situación de la legitimidad de la Administración pública en el caso espanool, empezando por las grandes reformas del sistema políticoadministrativo para terminar con lo relativo a la modemización de la Administración.

\section{Las grandes reformas del sistema político- administrativo: legitimidad y percepción de eficacia}

Las transformaciones del sistema político-administrativo no son sólo un dato del contexto de la Administración, sino que constituyen el cambio más radical que ha experimentado la Administración pública en España. Desde esta perspectiva la transición democrática, el desarrollo del Estado de bienestar, la descentralización y la integración europea son las principales reformas del sistema político-administrativo y han ejercido un notable impacto sobre las organizaciones públicas y sus formas de funcionamiento. Todas estas transformaciones fueron una especie de asignaturas pendientes, cambios aplazados, que se saldaron razonablemente en un breve periodo de tiempo y que supusieron la convergencia $\longrightarrow$ si se quiere la homologaciónde nuestro sistema político-administrativo con el de las restantes naciones de Europa. Muchas de las pautas de cambio que observara Rose en su Understanding Big Government (1984) y que se desarrollaron en otras naciones desde la segunda guerra mundial, en España se reprodujeron durante las décadas de 1970 a $1990^{4}$.

Los datos del cambio son de sobra conocidos pero merece la pena recordarlos.

Asi, por lo que respecta a la democratización, supuso un reforzamiento del proceso de profesionalización de los empleados públicos iniciado durante la década de 1960 . Al mismo tiempo, favoreció un modo de gestión de las políticas públicas más pluralista, y se limitó, en parte, el poder de la burocracia central.
Paralelamente, tuvieron lugar importantes cambios en el tamaño y el alcance funcional del Estado. Ello se tradujo en el crecimiento del gasto y el empleo público, así como en la expansión de los recursos invertidos en prestaciones sociales e intervención en la economía. Paralelamente se incrementó la presión fiscal, especialmente de los impuestos sobre los rendimientos del trabajo y capital. Uno de los impactos más importantes del crecimiento del Estado ha sido el aumento del grado de dependencia de las familias, tanto en lo relativo a ingresos directos de carácter regular como al consumo de bienes y servicios públicos (véase la tabla 1).

Otra de las transformaciones más significativas ha sido la descentralización. Ello se ha plasmado en el reconocimiento de la autonomía de los gobiernos subnacionales, la creación de Parlamentos en las Comunidades Autónomas y en una considerable transferencia de funciones hacia los gobiernos territoriales. De hecho, ha aumentado el tamaño de las Administraciones de estos gobiernos, tanto en lo que respecta al empleo como al gasto público -especialmente en las áreas de bienestar social-, a un ritmo superior al experimentado por el nivel central. Asimismo, se ha ido desarrollando un sistema de relaciones intergubernamentales sobrepuesto, en el que ha crecido la interdependencia de los gobiernos y la gestión intergubemamental de políticas y programas. La gestión intergubernamental, por su parte, ha sido desbordada por un tipo de gestión interorganizativa en la que están implicados, además de los tres niveles de gobiemo, organizaciones supranacionales y la sociedad civil.

Por último, la integración internacional ha favorecido la desregulación vía transposición de directivas comunitarias, así como las privatizaciones y la reconversión del sector público empresarial. Asimismo, se ha hecho sentir la europeización de las políticas públicas, desde la políica económica hasta terrenos más novedosos, como pueda ser la política de inmigración.

Obviamente, la magnitud, velocidad y simultaneidad de los cambios ha producido tensiones y desajustes, como son el desequilibrio entre responsabilidad fiscal y de gasto de las Comunidades Autónomas o el crecimiento del déficit o la deuda pública, por mencionar algunos de los aspectos más conocidos.

En cualquier caso, el sistema político-administrativo resultante goza -de acuerdo con los datos de opinión pública- de un razonable apoyo social en cualquiera de las dimensiones que se considere. Desde la más global -la creencia en la legitimidad de la democracia - hasta la interiorización del Estado de bienestar, pasando por la aceptación del Estado de las Autonomías o la integración europea.

Así, por lo que respecta a la democracia se ha arraigado la creencia de que el sistema democrático es preferible a cualquier 
otra forma de gobierno. El Estado de bienestar también es una realidad enraizada en términos de opinión. De las diversas formas de intervención se apuesta por el esquema clásico del Estado de bienestar basado en un modelo de equidad del tipo de igualdad de oportunidades, frente a la opción compensatoria o la liberal. Asimismo, se acepta el papel redistribuidor del Estado e incluso son más los ciudadanos que prefieren la opción de mejorar los servicios públicos, aunque ello suponga incrementar los impuestos, que la alternativa de reducir la presión fiscal a costa de disminuir las prestaciones sociales y los servicios públicos. Lo mismo puede afirmarse de la aceptación del Estado de las Autonomías. La opinión predominante es mantener la situación actual, seguida de la profundización en la descentralización, siendo minoritarias las posiciones más extremas. Por último, respecto a la construcción europea puede observarse también un elevado grado de apoyo, si bien con el tiempo se ha ido rebajando el euro-optimismo inicial de los españoles (véanse las tablas 2 a 5 ).

Tabla 1

Indicadores del cambio del sistema político-administrativo (1975-1995)

\begin{tabular}{|c|c|c|c|}
\hline & $1975 \%$ PIB & $1995 \%$ PIB & $\% 1995 \cdot \% 1975$ \\
\hline Gastos no financieros & 26.07 & 47.25 & 21.18 \\
\hline Gastos prestaciones sociales y bienes preferentes & 16.15 & 27.75 & 11.60 \\
\hline Presión fiscal & 21.35 & 35.34 & 13.99 \\
\hline Impuestos sobre las familias (renta y capital) & 4.86 & 12.79 & 7.83 \\
\hline Gastos públicos Adninistraciones territoriales & 2.45 & 11.57 & 9.12 \\
\hline \multirow[t]{2}{*}{ Ingresos fiscales Administraciones territoriales } & 1.70 & 4.62 & 2.92 \\
\hline & 1978 & 1995 & 1995-1978 \\
\hline \% de empleo público sobre población ocupada & 11.9 & 17.9 & 6.0 \\
\hline
\end{tabular}

\section{Tabla 2}

La legitimidad de la democracia

¿Con cuál de las siguientes frases está usted más de acuerdo?

La democracia es preferible a cualquier forma de gobierno

En algunas circunstancias un régimen autoritario, una dictadura puede ser preferible al sistema democrático

A las gentes como yo lo mismo nos da un régimen que otro

NS / NC

1980
$\%$

49

10

11

30
1985

$\%$

70

10

9

11

1995
$\%$
76
10
8
6

Fuente: Centro de Investigaciones Sociológicas y Demoscopia. 


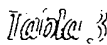

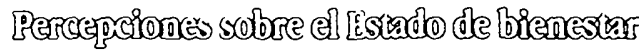

¿Cuãl de las siguientes frases se acerca más a su opinión personal?

El gobiemo es responsable del bienestar de todos y cada uno de los cildadaros y tiene la obligación de ąudartes a solucionar sus problemas

El gobiemo sólo es responsable del bienestar de los ciudadanos más desfarorecidos y tiene la obligación de ayudarles a solucionar sus problemas

Los ciudadanos son los verdaderos responsables de su propio bienestar y tienen la obligación de valerse por si mismo pam solucionar sus problemas

NS / NC

Dígame, por favor, hasta qué punto está usted de acuerdo con la siguiente frase: all Estado tiene la responsabilidad de reducir las diferencias entre las personas que tienen altos ingresos y las que tienen bajos ingresos.

Muy de acuerdo

De acuerdo

$\mathrm{Ni}$ de acuerdo ni en clesacuerdo

En desacuerdo

Muy en desacuerdo

NS / NC

Si el gobierno pudiera elegir entre bajar los impuestos o gastar más en servicios püblicos y prestaciones sociales, ¿qué cree usted que deberia hacer? (Nos referimos a la totalidad de los impuestos)

Gastar más en prestaciones sociales y servicios públicos, aunque esto signifique pagar más impuestos

Fuente: Centro de Investigaciones Sociológicas

\section{Trablo}

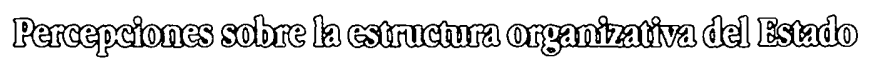

De las siguientes alternativas sobre la organización del Estado, digame con cuál está usted más de acuerdo:

IIn Fitado con un único Cobictno central sin autonomias

Un Estado con Comunidades Autónomas como en la actualicad

Lin Estado en el que las Comunidades Autónomas tengan mayor

autonumia que en la acrualidad

Un Estado en que se reconociese a las Comunidacles Autónomas la posibilidad de convertirse en Estados independientes

NS / NC

\section{4}

$\%$

29

31

20)

10

10
1990

$\%$

17

41

19

22

7

11

Fuente Centro de Invesugaciones Sociológicas 


\section{Tabla 5}

\section{Percepciones sobre la construcción europea}

En general ¿está usted a favor, bastante a favor, más bien en contra o muy en contra de los esfuerzos que se están haciendo para unificar Europa?
1986
1987
1991
1995

1988

1993

¿En su opinión, y en general, pertenecer a la Unión Europea ha sido para España una cosa buena, una cosa mala o una cosa ni buena ni mala?

$\begin{array}{lr}1986 & 43 \\ 1987 & 36 \\ 1988 & 49 \\ 1991 & 52 \\ 1993 & 18 \\ 1995 & 25\end{array}$

$\%$ a favor

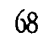

75

70

68

60

73

$\%$ bueno - malo

43

36

49

52

18

25

Fuente- Centro de Investigaciones Sociológıcas.

No obstante, también se ha de señalar que existen demandas de mejora respecto al funcionamiento de la democracia, o tensiones no resueltas, como el problema de la integración nacional o la atribución de impactos negativos para la economía española del proceso de integración en Europa.

Por otro lado, ha de destacarse que los gobiernos y las Administraciones públicas han hecho su contribución para dotar de eficacia al sistema político democrático. En este sentido, se puede observar una sensible mejora en lo que respecta a la percepción de los servicios públicos (véase la tabla 6). El grado neto de satisfacción ha aumentado en todos los casos respecto a 1992. Asimismo, este indicador resulta favorable en todos los servicios si se exceprúa la Administración de Justicia.

No obstante, se debe efectuar una matización —que ha de tenerse en cuenta cuando se realizan estudios de opinión en este ámbito- y que refuerza el sentido de la interpretación de estos datos. Así, cuando se pregunta a los consumidores de bienes y servicios públicos, el grado de satisfacción aumenta notablemente en casi todas las áreas de actuación incluida la propia
Administración de Justicia. Por otra parte, la percepción de los servicios tiende a ser mejor que la de las políticas.

En cualquier caso, se puede concluir que dos de las condiciones básicas para que la Administración pública pueda contar con legitimidad se cumplen: operar en el marco de un sistema político legitimo, de hecho las grandes reformas del sistema políico-administrativo cuentan con el apoyo social; y dotar de eficacia al sistema político, puesto que se ha producido una mejora en la percepción de los servicios públicos.

\section{La presunción de ineficiencia y el desafío de la legitimidad}

Sin embargo, las grandes reformas no han mejorado la imagen de la Administración, pues pervive un discurso social desfa- 


\begin{tabular}{|c|c|c|c|c|}
\hline \multicolumn{5}{|c|}{ Tabla 6} \\
\hline & $\begin{array}{c}1992 \\
\%\end{array}$ & $\begin{array}{c}1994 \\
\%\end{array}$ & $\begin{array}{c}1995 \\
\%\end{array}$ & $\begin{array}{c}\text { Diferencial } \\
1995-92^{6}\end{array}$ \\
\hline Enseñanza & 18 & 22 & $3 j$ & 17 \\
\hline Asistencia sanitaria en hospitales & -24 & -2 & 1 & 25 \\
\hline Asistencia sanitaria en ambulatorios & -28 & -2 & 1 & 29 \\
\hline Correos & -18 & 20 & 28 & 46 \\
\hline Teléfonos & ; & $\$ 1$ & 59 & 34 \\
\hline Gestión de las pensiones & -13 & -5 & 20 & 33 \\
\hline Policia & 0 & 4 & 14 & 14 \\
\hline Administración de justicia & -38 & -39 & -34 & 4 \\
\hline Servicios sociales & -12 & 0 & 19 & 31 \\
\hline Protección por desempleo & .33 & .23 & 13 & 46 \\
\hline Oficinas de la Administración pública en general & .26 & -26 & 7 & 33 \\
\hline Ferrocarriles & 1 & 24 & 35 & 34 \\
\hline Transporte aéreo & 17 & 32 & 43 & 26 \\
\hline Transporte urbano & 9 & 29 & 44 & 35 \\
\hline
\end{tabular}

Fuente: Centro de Investigaciones Socıologicas. Senes Históncas del CIS.

vorable. No resulta demasiado complicado identificar el estereotipo sobre la Administración pública. Una Administración sobre todo burocrática, lenta, complicada, opaca, ineficaz, autoritaria, desmotivada, alejada del ciudadano, desorganizada y despilfarradora. Asimismo, entre los lugares comunes se subrayan rasgos como la descoordinación y la existencia de duplicidades innecesarias, el exceso de funcionarios y cargos públicos, el desequilibrio entre impuestos pagados y servicios recibidos y la ineficiencia en comparación con el sector privado.

El análisis de Dízz Ramos a partir de estudios cualitativos y de una encuesta del CIS resulta bastante ilustrativo en cuanto a la comparación público-privado:

"En primer lugar, hay que destacar que la Administración Pública participa, con carácter genérico, de los rasgos de imagen que, hoy por hoy, caracterizan a la gestión pública frente a la gestión privada de servicios. La gestión pública aparece investida de rasgos negativos de imagen en todo lo relacionado con los valores de eficiencia y eficacia, por lo que la mayoría de los espanoles, un $50 \%$, considera que funcionan mejor los servicios en el sector privado que en el público, mientras que una escasa minoría del $16 \%$ opina lo contrario... La diferencia de imagen entre el funcionamiento de lo público y lo privado es contundente y refleja la persistencia de los tópicos negativos que se adscriben a la gestión pública. Así, cuando se fuerza una descripción de los rasgos que se pueden utilizar para caracterizar al sector público us. el privado, el único valor positivo que se asocia al sector público es su mayor preocupación social. Por el contrario, el resto de los calificativos testados se asocian en sentido negativo con lo público, que se considera más burocrático y mucho menos eficaz, bien organizado, competitivo, preocupado por la calidad de servicio, la satisfacción del cliente y la motivación y responsabilidad en el trabajo... Este trasfondo de opinión, que contrapone las bondades de la gestión privada frente a la pública, no puede ser más explícito: los tópicos tradicionales persisten y parece difícil que las Administraciones públicas, que representan la gestión por excelencia, no participen de ellos.

Este discurso social estereotipado podríamos denominarlo, parafraseando a LAUFER y BURLAUD (1988), la presunción de ineficiencia, en virtud de la cual los ciudadanos suelen atribuir efi- 
ciencia al sector privado mientras que en el caso de la Administración pública presuponen su ineficiencia. Esta pauta no es exclusiva de España y no faltan obras literarias y cinematográficas que permitan corroborar la resistencia al tiempo de estos estereotipos y su capacidad para cruzar las fronteras nacionales. LAUFER y BURLAUD explican el fenómeno por la opacidad de las organizaciones. Éstas son como cajas-negras especialmente vulnerables a las construcciones sociales. En el caso que nos ocupa, la opacidad de la caja-negra administración hace que se tienda a valorarla mediante una serie de estereotipos negativos, forjados a través de los siglos, incrustados en la cultura de las naciones, relativamente estáticos y difíciles de cambiar incluso aunque se tengan experiencias satisfactorias que siempre serán consideradas como algo excepcional.

La presunción de ineficiencia hemos de aceptarla como una constante difícil $\multimap$ imposible - de cambiar con independencia de que se pueda mejorar la imagen de una organización concreta o nivel de gobierno, conscientes de que la "Administración Públican -en mayúsculas y singular - es una construcción social tópica cuyo perfil de imagen tiende a ser negativo.

Más arriesgado es basar la formulación de la política de modernización en la presunción de ineficiencia. Los estereotipos sociales y los lugares comunes son conocidos en la literatura de políticas públicas como una de las fuentes de error en la definición de problemas y la formulación de las políticas (HoGwOOD y GuNN, 1988).

La interiorización del estereotipo es tal que no es extraño encontrar reformadores bien intencionados que frente a este discurso intenten mejorar la imagen de la Administración pública introduciendo medidas para simplificar y dotar de mayor agilidad a los procedimientos; mejorar la comunicación con los ciudadanos introduciendo un lenguaje más asequible y nuevos sistemas de información y comunicación; la creación de ambientes agradables en la distribución de espacios en las oficinas públicas, la identificación de los empleados públicos y su formación en la atención al público; la reducción de tiempos de espera, la modificación de horarios y muchas otras medidas que disminuyan los costes de transacción entre el ciudadano y la Administración. Algunos reformadores más radicales intentarán también mejorar los sistemas de coordinación, delimitar claramente las competencias, reducir el número de órganos y empleados públicos e incrementar la eficiencia mediante la introducción de nuevas formas de gestión que hagan funcionar la Administración como una empresa.

Sin embargo, todo ello no cambiará la presunción de ineficiencia, más bien al contrario, cuanto más radical sea esta actuación, en mayor medida confirmará el estereotipo inicial ${ }^{7}$. No quiero decir con ello que no sea aconsejable reducir los costes de transacción entre Administración y ciudadano, o que no se deba perseguir la eficiencia. Antes al contrario. Lo que se quiere indicar es que una política de modernización orientada exclusivamente a dar respuesta al estereotipo es una suerte de política populista, que a lo que contribuye en última instancia es a reafirmar la presunción de ineficiencia inicial.

El problema no reside en la presunción de ineficiencia. Esta forma parte de nuestra cultura político-administrativa. Ha estado ahí durante mucho tiempo y ello no ha supuesto el desmantelamiento de la Administración. Es cierto que en un momento en el que se cuestiona lo público resulta más incómoda, pero el problema está en otro lugar: en la legitimidad.

De hecho, en términos de opinión pública se observan críticas respecto de la distancia entre los valores socialmente aceptados sobre cómo debe ser el comportamiento de lo público y la percepción de las Administraciones públicas, especialmente en el caso de la Administración central ${ }^{8}$. El que se considere a la Administración lenta es un mal menor y no va a cambiar esta percepción aunque se reduzcan los tiempos de respuesta de los trámites administrativos. El problema es que se considere que las Administraciones públicas son arbitrarias; que no se identifique en sus actuaciones un modelo de equidad; o se estime que no responden a las necesidades y demandas de los ciudadanos, de tal forma que se crea que su actuación está guiada por criterios espurios generalmente de carácter político que nada tengan que ver con el interés general. Este otro discurso es más problemático pues afecta a valores esenciales en los que se basa la legitimidad de la Administración.

Pero al margen de las interpretaciones que se puedan ofrecer al respecto ", la cuestión es en qué medida la modernización de la Administración, y más en concreto lo que se conoce como nueva gestión pública (NGP), contribuye a la legitimidad de la Administración.

\section{A modo de conclusión: la nueva gestión pública y la legitimidad de la Administración}

La relación de opciones e instrumentos de política pública a las que con distinta intensidad recurren los gobiernos de las naciones desarrolladas para la reforma de la Administración pública son conocidos y han sido analizados por diversos autores ${ }^{10}$. Menos énfasis se suele poner en los valores subyacentes a estas opciones. Pout (1995), tras revisar el discurso de políticos de diversas naciones desarrolladas comprometidas en políticas de 
modemización, identifica una primera relación de efectos esperados de las reformas. En este sentido, la NGP deberá: producir mayor ahorro, mayor eficiencia, elevar los niveles de los servicios públicos, incrementar la autonomía de los gestores de servicios y mejorar la receptividad del personal de la Administración hacia los usuarios de los servicios".

Toda esta lista de efectos esperados - caso de que efectivamente lograran alcanzarse con las políticas de modemización ${ }^{12}$ refuerzan lo que al principio de este trabajo se denominó la legitimidad por rendimientos de la Administración. Los objetivos arriba mencionados giran principalmente en tomo a los valores de calidad, eficacia y eficiencia. Ello es sin duda muy importante pues, como vimos, la misión principal de las Administraciones públicas es dotar a través de sus rendimientos de eficacia al sistema político. Sin embargo, esto no es suficiente para mejorar la legitimidad de la Administración. Veamos esta cuestión más detenidamente.

Calidad, eficacia y eficiencia son tres criterios importantes a la hora de valorar el rendimiento tanto de una organización pública como privada. Sin embargo, cuando se trata de organizaciones públicas se ha de añadir otro criterio de evaluación del rendimiento: la equidad. Al afirmar esto no se está prejuzgando un modelo de equidad determinado - igualdad de oportunidades, compensatorio o de mercado- sino simplemente se subraya que a las organizaciones públicas también se les reclama que sean justas. Por otra parte, la prioridad de estos criterios sigue un orden peculiar: equidad, calidad, eficacia y eficiencia (Crompton y LaMB, 1986). Ya que se puede ser muy eficiente y al mismo tiempo injusto. La eficiencia en lo público ha de considerarse una vez seleccionado un modelo de equidad, esto es, como las formas de gestión más eficientes una vez optado por un estándar de equidad ${ }^{13}$.

Por otra parte, es muy difícil -salvo en el caso de algunas políticas - que se pueda llegar a un consenso entre los actores involucrados en cada política o servicio sobre: qué es justo a la hora de repartir costes y beneficios; cuáles son las expectativas de beneficio a satisfacer y con qué prioridad; cuáles son los impactos deseados; y cuáles son los verdaderos costes a considerar. Así, por ejemplo, las mejoras de la calidad con frecuencia terminan traduciéndose en juegos de traslación de costes de un actor a otro. Por otra parte, el terreno del consumo colectivo de servicios públicos está abonado para generar un tipo de conflictos en función de la posición individual -más que de la clase social de pertenencia - ante el consumo colectivo que hace que las actuaciones públicas siempre dejen a sectores parcialmente satisfechos e insatisfechos, reconstruyéndose para cada caso un grupo de ganadores y perdedores específico en el que la posición individual varía de servicio en servicio.
Pero más importante que todo esto es « peso que tienen los componentes de carácter institucional en la legitimidad de la Administración. La legitimidad por rendimientos es importante, pero más lo es la legitimidad institucional ${ }^{14}$. Ello se debe, en mi opinión, a una suerte de mecanismo de control social derivado de las dificultades de revelación de preferencias en el ámbito de los bienes y servicios de carácter público, así como a otros factores vinculados a las formas de funcionamiento de los sistemas político-administrativos. Así, y parafraseando a LIPSET, una legitimidad por rendimientos alta no permite compensar una legitimidad institucional baja o, dicho de otra manera, la capacidad de los rendimientos por sí solos para mejorar un déficit de legitimidad institucional es limitada. Por supuesto que un bajo rendimiento de forma prolongada acabaría por arruinar la mejor legitimidad institucional.

La NGP está desde esta perspectiva demasiado sesgada al rendimiento y ello no deja de plantear problemas desde el punto de vista de la legitimidad de la Administración. Sin embargo, hay otro punto que también merece la pena destacar: los riesgos de la maximización de valores. Al poner el énfasis en la eficiencia, la eficacia y la calidad se corre el riesgo de que se produzcan pérdidas en otros valores en los que se sustenta la justificación de la Administración pública. La cuestión reside en las dificultades de maximización de un valor sin que sea a costa de la posición relativa de los restantes valores.

Ello nos lleva de lleno al terreno de los efectos no esperados de la NGP. Aquí nuevamente se nos aparece la noción de equidad, más todos los valores en los que se sustenta la legitimidad institucional, viejos aspectos de lo público que aparecen ante el modernizador como dinosaurios revividos: el modelo racional-legal de dominación con cuadro administrativo burocrático, el Estado de bienestar y la idea de la responsabilidad administrativa.

Una NGP muy radical que choque con los componentes instirucionales de la legitimidad de la Administración corre el riesgo de deslegitimarse. La NGP tendrá que someterse a la misma maldición de los fallos del Estado. La teoría de los fallos del Estado decía que no basta con que se produzca un fallo del mercado para que se justifique la intervención del Estado; también habrá de demostrarse que la actuación pública tal y como se comporta el sistema político y la Administración en la realidad es capaz de mejorar la situación. La NGP tendrá que probar que no basta con que se intente corregir los fallos del Estado, sino que la corrección de estos fallos, tal y como se produce en la realidad, tenga los impactos esperados y no genere impactos no esperados que afecten a valores esenciales del sistema politico-administrativo democrático, pues en ese caso más valdría la enfermedad que el remedio. El tiempo nos dirá si la NGP y las políticas de modernización fueron capaces de superar esta maldición. 
- Universidad Complutense de Madrid. Instituto Universitario Ortega y Gasset.

' El autor quiere agradecer los comentarios y la información proporcionada para la elaboración de este arículo a los profesores Isabel Bazaga, Eloisa del Pino, Juan Antonio Ramos, Manuel Tamayo y Manuel Villoria.

2 Para una critica a LIPSET véase BEETHam (1991).

" Con el inconveniente de que ante cada política o servicio concreto cada actor social involucrado fácilmente entiende cosas distintas sobre lo que es justo, tiene expectativas de beneficio diferentes, persigue impactos distintos y calcula los -verdaderos. costes de forma también diferente.

4 Por lo que respecta al sistema politico-administrativo -como en tantos otros aspectos de carácter sociał - se avanzó en el viejo deseo del pensamiento ilustrado del siglo XVIII de eponerse al unisono con los paises más civilizados de Europa. (SA. RRALH, 1979). Desde mi punto de vista estos cambios constituyen la modemización con mayúsculas de la Administración pública.

- Diferencia entre \% de entrevistados que valora funcionamiento -muy o bastante satisfactorio, y \% que valora poco o nada satisfactorio.

- El diferencial 1995-92 ha sido calculado mediante la resta del grado neto de satisfacción de 1995 al de 1992.

' Por expresarlo gráficamente -fijese si sobrarán funcionarios que cada politico que llega nuevo dice que va a reducir su número.

${ }^{8}$ Para el caso espaǹol véase DEL PINo (1997) donde apora abundante información empírica tanto cuantitativa como cualitativa al respecto.

${ }^{2}$ Este discurso puede ser explicado desde varias ópticas. Asi, por lo que se refiere a la percepción desfavorable de la Administración central, existe lo que podriamos denominar la ley de hierro de la visibilidad, en virtud de la cual los ciudadanos prefieren aquello que pueden tocar y tienden a desconfiar de aquello que no ven. La ley de hierro de la visibilidad es un poderoso argumento a favor de la descentralización, pero sobre todo plantea el problema de la legitimidad de los distintos niveles de gobiemo, ya que llevada a sus últimos extremos viene a afirmar que los problemas de legitimidad aumentan conforme ascendemos en el nivel de gobierno. Asimismo, exis- te una tendencia a que las instituciones administrativas sean peor valoradas que los rendimientos -la propia opacidad de la caja negra administración que senalaran LaU. FER y BURLAUD, unida a la frecuente carencia de políticas activas de comunicación por parte de las organizaciones públicas, explican en parte este fenómeno, así como la orientación ciudadana a preocuparse más por el salario directo o indirecto que pueda proporcionar el sector público que por el funcionamiento de la Administración-. Otras explicaciones tienen que ver con los primeros sintomas de la crisis del Estado de bienestar y de las instituciones políticas y administrativas y la transformación de los valores respecto de lo público. Así, al igual que no se cuestiona la democracia, pero si su funcionamiento operativo, también se empieza a cuestionar la forma de operar de los gobiernos y las Administraciones públicas y los defectos más sobresalientes de la burocratización, al tiempo que comienzan a adquirir cada vez mayor relevancia las demandas de profundización de la democracia y del desarrollo de la Administración democrática. Por último, se está haciendo referencia a un espacio difuso, el de la Administración pública, en muchos casos difícil de deslindar en términos de opinión del gobierno, la politica, los partidos, lo público, etc., de tal forma que existe una fuerte contaminación de unas instituciones con otras.

10 Véanse entre otros BARZELAY (1992), POLITT (1993), MÉNY y W/RIGHT (1994), VILLO RIA (1996).

"Algún malintencionado pensaría que se trata de una lista de objetivos loables que casan bien con la presunción de ineficiencia.

12. Como señalara Pourrr (1995), no deja de llamar la atención que tras haber puesto tanto énfasis la NGP en la evaluación; el número de evaluaciones de los impactos de la implantación de la NGP disten bastante de ser abundantes.

${ }^{14}$ Es más eficiente asignar recursos de formación para el empleo a jóvenes titulados de clase media que a jóvenes expulsados del sistema escolar. Si se opta por un modelo de equidad compensatorio la cuestión de la eficiencia consistirá en cómo gestionar los recursos para conseguir producir empleo a través de la formación a jóvenes expulsados del sistema escolar.

1" Aunque sobre este extremo existen variaciones dependiendo del nivel de gobierno del que se trate, la arena de politica pública y el tipo de bien y servicio.

Bibliografia

BARZELAY, M. Breaking through Bureaucracy: A New Vision for Managing in Government. University of Califomia Press. Berkeley. 1992.

BEETHAM, D. The Legitimation of Power. MacMillan. Londres. 1991.

CROMPTON, J.L. y LAMB, Ch.W. Marketing Govermment and Social Services. Wiley and Sons. Nueva York. 1986.

Del PINO, E. cLa imagen de la Administración pública, ponencia presentada al III Congreso de Ciencia Politica y de la Administración. Salamanca. 1997.

Dfez Ramos, M. La comunicación. Marco sociológico. Mimeo. s.l., s.a.

GARCla LOPEZ, J. y ALVIRA, F. .Personal al servicio de las administraciones públicas, en Papeles de Economia. Madrid. 1996. n. $96-97$.

HocwOOD, B.W. y GUNN, L.A. Policy Analysis for the Real World. Oxford University Press. Oxford. 1988.

LAUFER, R. y BURLAUD, A. Dirección priblica: gestión y legitimidad. INAP. Madrid. 1989.
LIPSET, S.M. El bombre politico. Las bases sociales de la politica. Tecnos. Madrid. 1987. MÉNY, Y. y WRIGHT, V. La riforma amministrativa in Europa. Il Mulino. Bolonia. 1994. Poust, Ch. Justification by Works or by Faith? Evaluating the New Public Management, en Evaluation. Sage. 1995. Vol. 1. n. ${ }^{\circ} 2$.

ROSE, R. Understanding Big Government. Sage. Londres. 1984.

SARRARH, J. La España llustrada de la segunda mitad del siglo XVIII. Fondo de Cultura Económica. México. D.F. 1979.

Starung, G. Managing the Public Sector. Dorsey Press. Homewood. 1986.

VAUE, V. LLa hacienda pública de la democracia espanoolao, en Papeles de Economia. Madrid. 1996. n. ${ }^{\circ}$ \%6-97.

VนORIs, M. La modernización de la Administración como instrumento al servicio de la democracia. INAP-BOE. Madrid. 1996.

WEBER, M. Economia y sociedad. Fondo de Culura Económica. México. D.F. 1979. 